\title{
Inventario de Ideación Suicida Positiva y Negativa (PANSI): Propiedades Psicométricas en Universitarios Peruanos
}

\author{
Positive and Negative Suicide Ideation (PANSI) Inventory: Psychometric Properties \\ in Peruvian University Students
}

\author{
Nikolai Martin Rodas-Vera ${ }^{1}$, Ronald Toro ${ }^{2}$ y Pablo Ezequiel Flores-Kanter ${ }^{3}$
}

\begin{abstract}
Resumen
Es necesario contar con instrumentos que evalúen la ideación suicida, dado que es uno de los primeros factores que conllevan al suicidio. La presente investigación realizó un proceso de adaptación del Inventario de Ideación Suicida Positiva y Negativa (PANSI) para que pueda ser empleado en universitarios peruanos. Se estudiaron dos muestras, una antes y otra durante la pandemia por COVID-19. A nivel metodológico, se siguió un procedimiento racional con jueces expertos y con sujetos de la población para conseguir la equivalencia cultural y conceptual del instrumento; mientras que, en el nivel estadístico, se probaron tres modelos teóricos, unidimensional, ortogonal y oblicuo. El modelo de dos factores correlacionados reveló mejores índices de ajustes para ambas muestras, con niveles óptimos de confiabilidad. Se concluye en una nueva versión del PANSI y se discuten sus bases teóricas para mejorar las inferencias a partir de este instrumento.
\end{abstract}

Palabras clave: PANSI, ideación suicida, propiedades psicométricas, universitarios peruanos, COVID-19

\begin{abstract}
It is necessary to rely on tools that evaluate suicidal ideation, given that it is one of the first factors that lead to suicide. This research performed an adaptation process of the Positive and Negative Suicidal Ideation (PANSI) so that it can be used in Peruvian university students. Two samples were studied, one before and one during the COVID-19 pandemic. At the methodological level, a rational procedure was followed with expert judges and individuals from the population to achieve cultural and conceptual equivalence of the tool; while, at the statistical level, three theoretical models were tested: unidimensional, orthogonal and oblique. The two-factor correlated model revealed better fit indexes for both samples, with optimal levels of reliability. We completed a new version of the PANSI and discussed its theoretical basis for improving inferences from this tool.
\end{abstract}

Keywords: PANSI, suicidal ideation, psychometric properties, Peruvian university students, COVID-19

Los autores agradecen el apoyo de la Universidad César Vallejo por el apoyo a la publicación del presente artículo.

\footnotetext{
${ }^{1}$ Psicólogo, Docente Investigador. Universidad César Vallejo, Lima, Perú. Tel.: (511)959137153. Correo: nikolairodas@gmail.com, nrodas@ucv.edu.pe

${ }^{2}$ Psicólogo Clínico, PhD. en Salud, Psicología y Psiquiatría. Docente Investigador. Universidad Católica de Colombia. Grupo GAEM, Bogotá, Colombia.

${ }^{3}$ Magister/Becario CONICET/ a: Consejo Nacional de Investigaciones Científicas y Técnicas (CONICET), Argentina; b: Instituto Tecnológico de Buenos Aires (ITBA); c: Centro de Bioética de la Universidad Católica de Córdoba, Argentina.

Revista Iberoamericana de Diagnóstico y Evaluación - e Avaliação Psicológica. RIDEP · No60 · Vol.3 · 27-39 2021

ISSN: 1135-3848 print /2183-6051online
} 


\section{Introducción}

El suicidio es reconocido como un problema de salud mental que afecta a la población mundial (Organización Mundial de la Salud [OMS], 2006), aparece con mayor prevalencia en países de medianos o bajos ingresos económicos (OMS, 2019), es la tercera causa de muerte en jóvenes de 15 a 19 años (OMS, 2019) y hasta hace poco fue la segunda causa entre los 15 y los 29 años (OMS, 2014).

El suicidio genera un impacto negativo en las emociones de familiares, amigos y pareja, generando desconcierto, culpa y duelos alterados (Gómez, 2017) y complicados (Quagliata, 2015); pero sus consecuencias van más allá del nivel interpersonal. Se estima que, a causa del suicidio, se pierden millones de dólares en todo el mundo (Pompili, 2012) y que en América es la quinta causa más importante de años de vida perdidos por discapacidad y mortalidad (Organización Panamericana de la Salud, 2018).

En Latinoamérica hay evidencias de suicidios e ideación suicida en diferentes grupos sociales y minoritarios. Por ejemplo, en lesbianas, gais, transexuales y bisexuales (LGTB; Tomicic et al., 2016) y en zonas rurales (Chaparro-Narváez, Díaz-Jiménez, \& Castañeda-Orjuela, 2018). Un grupo de interés es el de los universitarios (Carmona et al., 2017), en el que se observa una importante prevalencia de ideación suicida (Calvo, Sánchez, \& Tejada, 2003; DenisRodríguez, Barradas, Delgadillo-Castillo, DenisRodríguez, \& Melo-Santisteban, 2017; González, Medina, \& Ortiz, 2016; Sánchez, Cáceres, \& Gómez, 2002).

En el Perú, la salud mental está considerada como prioridad sanitaria y se ha planteado la necesidad de desarrollar investigaciones científicas sobre el suicidio (Ministerio de Salud, 2018), teniendo como criterio principal que el suicidio es prevenible (OMS, 2014). En este contexto, esta investigación pretende contribuir a dilucidar el complejo problema del suicidio, por medio de una de sus variables influyentes, como es la ideación suicida.

El problema del suicidio llama la atención en esta época, donde la OMS (2020a) declaró pandemia al coronavirus de tipo 2 (COVID-19) que causa el síndrome respiratorio agudo severo
(SRAS-CoV-2) (OMS, 2020b), generando que muchos países ingresen en cuarentena, lo que en Perú se produjo desde el 15 de marzo del 2020 hasta el 1 de julio del mismo año. Desde diciembre del 2019, fecha en la que apreció el COVID-19, países europeos (Ozamiz-Etxebarria, DosilSantamaria, Picaza-Gorrochategui, \& IdoiagaMondragon, 2020), asiáticos (Wang, et al 2020), así como latinoamericanos (González-Jaimes, Tejeda-Alcántara, Espinosa-Méndez, \& OntiverosHernández, 2020; Johnson, Saletti-Cuesta, \& Tumas, 2020) y específicamente el Perú (Mejia et al., 2020), han revelado graves problemas de salud mental, relacionados a ansiedad, depresión, estrés y pensamientos fatalistas asociados al COVID-19.

Todos estos trastornos y síntomas, antes mencionados, probablemente ocasionados por la soledad y el estrés de la cuarentena (Steenblock et al., 2020), pueden hacer que el suicidio se convierta en una pandemia psicológica global (Thakur \& Jain, 2020), de hecho, ya se han presentado casos de suicidio por COVID-19 (Mamun \& Griffiths, 2020) y la ideación suicida es uno de los indicadores que más se relaciona con la ansiedad por COVID-19 (Lee, 2020). Pero a pesar de que el riesgo de suicidio se ha podido incrementar (Killgore et al., 2020; Sher, 2020) y que, en el Perú, de 2466 encuestados, el 9\% manifestó que podría suicidarse si se contagiara con COVID-19 (Mejia et al., 2020), en este país la ideación suicida como tal aún no ha sido investigada durante la pandemia.

Es pertinente señalar algunas diferencias conceptuales alrededor del término que ocupa este estudio. El suicidio en sí es un acto autoinfligido e intencional con conocimiento o expectativa previa de la fatalidad (Baader, Urra, Millan, \& Yáñez, 2011; Goldney, 2008). En cambio, la ideación suicida, corresponde al pensamiento de comportamiento autoagresivo con intento suicida variable, pero sin letalidad (Goldney, 2008). Puede existir una ideación egosintónica, cuando se presentan sensaciones positivas relacionadas al pensamiento; o una ideación egodistónica, cuando se experimentan sensaciones desagradables, como culpa o vergüenza (Gómez, 2012). Por otro lado, pueden presentarse pensamientos pasivos (querer estar muerto) o pensamientos activos (pretender asesinarse a sí mismo) (Posner, Oquendo, Gould, Stanley, \& Davies, 2007). 
Por otro lado, el intento suicida es una conducta autolesiva pero no fatal a menudo con el propósito de movilizar ayuda (Goldney, 2008), mientras que el riesgo suicida hace referencia a la alta probabilidad de ocurrencia del desenlace fatal, integrada por la intención suicida (deseos de morir y elaboración de un plan para quitarse la vida), el nivel de letalidad del método, conocimientos sobre formas de autodestrucción y la inexistencia de un sistema de apoyo social (Beck, Kovacs, \& Weissman, 1979). Finalmente, la ideación suicida, unida a la desesperanza y el pensamiento rígido, entre otros, forman parte de los factores psicológicos en la evaluación del riesgo suicida (Gómez, 2012).

En base a esta revisión conceptual, se deduce que la ideación suicida es la variable que inicia el camino hacia el suicidio. Así, lo habían propuesto Beck, Kovacs y Weissman (1976, en Gómez, 2012), quienes señalaban que existía un continuo de suicidalidad conformada por las ideas suicidas, los intentos suicidas y el suicidio en sí. Del mismo modo Villalobos-Galvis (2010), indicaba que el espectro suicida empieza en la ideación, continuando por las alternativas y llegando al suicidio. Esta es una razón más para investigar la ideación suicida.

Para poder evaluar la ideación suicida es necesario contar con instrumentos adecuados. Entre los instrumentos más usados en Perú, se encuentra la Escala de Riesgo Suicida de Plutchik (Plutchik, Van Praag, Conte, \& Ricard, 1989; Santana-Campas \& Telles, 2018), cuyas versiones adaptadas, además de la ideación suicida, presentan las dimensiones de sentimiento de inutilidad, desesperanza y factores sociales; la Escala de Ideación Suicida de Beck (Beck, Brown, \& Steer, 1997; Beck, Kovacs, \& Weissman, 1979), que evalúa el deseo suicida activo, planes para el suicidio y el deseo suicida pasivo; y la Escala de Ideación Suicida de Roberts (Roberts, Roberts, \& Chen, 1998), que contiene ítems sobre pensamientos, deseos de estar muerto y planes para asesinarse.

Osman, Gutierrez, Kopper, Barrios y Chiros (1998) desarrollaron el Inventario de Ideación Suicida Positiva y Negativa (PANSI) en universitarios, encontrando dos factores. El primero, ideación suicida negativa, mide la frecuencia de pensamientos negativos en torno a la vida, que podría llevar al suicidio; es decir, funciona como un factor de riesgo. El segundo, ideación suicida positiva, mide la frecuencia de pensamientos positivos acerca de la vida, como entusiasmo, eficacia, esperanza, que funcionaría como un amortiguador frente al suicidio; es decir, consistiría en un factor protector ante el suicidio. Ambas dimensiones arrojaron adecuados índices de confiabilidad y validez en base a la relación con depresión y desesperanza.

Los análisis factoriales del PANSI han reportado esta estructura teórica bidimensional. En la versión original, Osman et al. (1998), encontró dos dimensiones correlacionadas con análisis factorial exploratorio y análisis factorial confirmatorio, comparado con una solución unidimensional no viable. En una revisión posterior con adolescentes psiquiátricos se confirmó la misma estructura original (Osman et al., 2002; Osman et al., 2003), confirmando que estos dos factores son correlacionados y que distingue adecuadamente a personas que intentan suicidarse. Luego con muestras multiétnicas, se confirmó el modelo de dos factores del PANSI con indicios de variaciones entre las respuestas de los participantes, un aspecto que sugiere verificar en los contextos particulares las propiedades psicométricas del instrumento (Muehlenkamp, Gutierrez, Osman, \& Barrios, 2005).

Desde entonteces, el PANSI ha sido usado extensivamente reportándose una estructura factorial igual a la original. Por ejemplo, en Nigeria mediante análisis factoriales exploratorios y confirmatorios encontraron una solución aceptable (Comparative Fit Index $[\mathrm{CFI}]=0.974$; Root Mean Square Error of Approximation $[$ RMSEA] $=0.040$ ), y correlaciones significativas con medidas depresivas y calidad de vida (Aloba, Adefemi, \& Aloba, 2018). En Malasia fueron reportados indicadores de una favorable estructura bidimensional oblicua (Sinniah et al., 2015), así mismo en China se halló una adecuada capacidad predictiva del intento suicida a un año, elevada consistencia interna y test retest (Chang, Lin, Chou, Ma, \& Yang, 2009).

En Latinoamérica, el PANSI fue adaptado al español en adolescentes y universitarios colombianos por Villalobos-Galvis (2010), quien encontró las mismas dimensiones mediante Análisis Factorial Exploratorio, adecuada 
confiabilidad y validez convergente y divergente con variables de depresión, autoestima, desesperanza y otra medida de ideación suicida. Posteriormente, Avendaño-Prieto, Pérez-Prada, Vianchá-Pinzón, Martínez-Vaquero y Toro (2018), analizaron el PANSI en adolescentes colombianos desde la Teoría de Respuesta al Ítem y el Modelo Rasch, eliminando uno de los ítems (¿Te sentiste tan triste por tu relación con alguien importante, que quisiste estar muerto?). No obstante, los autores no repararon en que este ítem mide ideación suicida pasiva (Beck et al., 1979; Posner et al., 2007), ya que denota el querer estar muerto, pero no la intención de matarse, tal vez por ese motivo resultó un ítem deficiente. Así que para la investigación que se presenta a continuación, este ítem fue transformado para que revele ideación suicida activa, dado que los demás apuntan hacia esta forma de expresar los pensamientos suicidas.

Es pertinente aclarar la teoría en la que se basa el PANSI. En primer lugar, parte de un modelo cognitivo, porque los ítems expresan el pensamiento o deseo de terminar con la propia vida (Brown \& Beck, 2009, en Toro-Tobar, Grajales-Giraldo, \& Sarmiento-López, 2016; Rush \& Beck, 1978; Toro-Tobar et al., 2016). Este modelo plantea tres pilares para entender la ideación suicida (Rush \& Beck, 1978). En primer lugar, la triada cognitiva (visión negativa sobre uno mismo, los demás y el futuro); en segundo lugar, los esquemas cognitivos; y, en tercer lugar, los errores cognitivos. Asimismo, tiene que ver con un modelo de ideación suicida activa, dado que los pensamientos intencionales de acabar con la existencia (Beck, et al, 1979; Posner, et al, 2007) se pueden encontrar reflejados en los reactivos de la dimensión ideación suicida negativa.

Finalmente, aunque si bien la ideación suicida puede entenderse desde un marco psicopatológico (Toro-Tobar et al., 2016) y neuroendocrinológico producto del estrés (Steenblock et al., 2020), la existencia de la dimensión ideación suicida positiva, puede brindar una redefinición de sus bases teóricas. Así, se podría pensar en el enfoque de la psicología positiva (Alarcón, 2009; Seligman, 2017; Seligman \& Csikszentmihalyi, 2014), debido a sus indicadores de confianza en las capacidades, esperanza en el futuro, sentimientos de alegría, sentido de vida y autodeterminación.

Por todo lo expuesto, se planteó analizar las propiedades psicométricas del PANSI adaptado a universitarios peruanos, evaluando evidencias de validez y confiabilidad antes y durante la pandemia por COVID-19. Los hallazgos serán importantes desde un punto de vista teórico para evaluar la estructura de dos factores correlacionados en esta nueva población; y desde un punto de vista práctico, dado que podrá ser usado como parte de una batería de instrumentos en evaluaciones clínicas.

\section{Método}

\section{Muestra}

Muestra 1. Estuvo compuesta por 306 universitarios de Lima, cuyas edades oscilaron entre los 17 y los 32 años $(M=22.5)$, donde los hombres representaron el 52.6\%. Esta primera recolección de datos se produjo alrededor de los seis primeros meses del 2019.

Muestra 2. Estuvo compuesta por 207 universitarios de diversas regiones del Perú, que respondieron el instrumento mediante formulario Google durante los primeros cuatro meses de la pandemia, con edades entre los 15 y los 53 años $(M=22.7)$, donde las mujeres representaron el $80.7 \%$; $87.9 \%$ personas declararon no ser pacientes de COVID-19, 7.7\% ser pacientes asintomáticos y $4.3 \%$, pacientes recuperados.

\section{Instrumento}

Fue empleada la versión colombiana (Villalobos-Galvis, 2010) del Inventario de Ideación Suicida Positiva y Negativa (PANSI, Osman et al., 1998), que como se mencionó, presentó adecuadas propiedades psicométricas. $\mathrm{La}$ primera dimensión, ideación suicida negativa $(\alpha=.93)$, contiene ocho ítems, con indicadores como: "¿Pensaste en matarte porque no tenías esperanza en el futuro?”, “ ¿Te sentiste tan triste por tu relación con alguien importante, que quisiste estar muerto?". La segunda dimensión, ideación suicida positiva $(\alpha=.836)$, contiene seis ítems, con indicadores como: " $¿ T e$ sentiste alegre porque te estaba yendo bien en el colegio o en el trabajo?”, “Sentiste que valía la pena vivir la 
vida?". Las opciones varían desde 0 (nunca) y 4 (siempre).

\section{Procedimiento}

Siguiendo las directrices internacionales sobre la adaptación de los tests (Muñiz, Elosua, \& Hambleton, 2013), se establecieron procedimientos para lograr el máximo nivel de equivalencia cultural y conceptual. En primer lugar, sobre la equivalencia cultural, el PANSI, contenía un ítem referido al colegio o al trabajo: " ¿Te sentiste alegre porque te estaba yendo bien en el colegio o en el trabajo?". Así, considerando que la población objetivo era universitaria, se prefirió cambiarlo por " ¿Te sentiste alegre porque te estaba yendo bien en la universidad?" eliminando la referencia al trabajo, puesto que no todos los universitarios trabajan. Además, considerando que los ítems sobre ideación suicida negativa presentan referencias explícitas al suicidio (e.g. "¿Pensaste en matarte porque no tenías esperanza en el futuro?”), se empleó jueces expertos en contenido y sujetos pertenecientes a la población (Martínez, Hernández, \& Hernández, 2014), a quienes se les realizó una breve entrevista sobre el impacto de los ítems. Los jueces fueron tres psicólogas clínicas, una de ellas experta también en evaluación psicológica, quienes señalaron la susceptibilidad que podrían ocasionar estos ítems en algunas personas, especialmente en los más jóvenes. Por otro lado, los sujetos de la población entrevistados fueron 10 universitarios de ambos sexos, donde seis coincidieron con las reacciones negativas que podrían ocasionar los ítems. Realizando un cruce de información, se decidió cambiar las referencias de "matarse" de cada ítem del factor ideación suicida negativa, indicando en las instrucciones del test: "En algunas preguntas encontrarás un espacio en blanco: el cual debe ser reemplazado por 'terminar con tu vida'". Así, por ejemplo, el ítem "Pensaste en matarte porque no pudiste hacer algo que era muy importante en tu vida", fue reemplazo por "¿Pensaste en porque no pudiste hacer algo que era muy importante para ti?". Esto fue aceptado por los jueces de contenido y los sujetos de la población entrevistados. Además, cuando se presentaba el instrumento, se conservaba por completo el nombre del instrumento y se informaba a los evaluados sobre el tema de la investigación.

En segundo lugar, se evaluó la equivalencia conceptual, donde se existieron dos consideraciones. Primero, como la adaptación cultural produjo cambios en el fraseo de los ítems, se tuvo cuidado de que esos cambios no modifiquen el indicador de cada ítem, respetándose así su esencia teórica, lo que fue examinado mediante entrevistas a los jueces expertos. Segundo, conociendo que AvendañoPrieto et al. (2018), hallaron el mal funcionamiento del ítem: " ¿Te sentiste tan triste por tu relación con alguien importante, que quisiste estar muerto?", se convino en analizar teóricamente este ítem antes de aplicar el instrumento y como es un ítem que mide ideación suicida pasiva (Beck et al., 1979; Posner et al., 2007), fue transformado para que revele ideación suicida activa, dado que los demás apuntan hacia esta forma de expresar los pensamientos suicidas. Así, este ítem quedó así: “¿Te sentiste tan triste por tu relación con alguien importante, que quisiste _? ?. De esta manera, todos los ítems quedaron como indicadores de ideación suicida activa.

Terminado el proceso metodológico de adaptación, se aplicó el instrumento antes de la pandemia por COVID-19 y durante la pandemia. En este segundo caso, el instrumento fue elaborado en formulario Google y enviado a estudiantes universitarios.

\section{Análisis de datos}

En primer lugar, se llevaron a cabo análisis exploratorios preliminares de los datos, con el objetivo de verificar la normalidad univariada y multivariada de las puntuaciones obtenidas. Posteriormente, los puntajes de los ítems del PANSI fueron modelados usando el análisis factorial confirmatorio (CFA, Ondé, 2020), técnica estadística que forma parte del sistema de ecuaciones estructurales (Sánchez-Martí, Muñoz, \& Ion, 2019). Dado que se trabajó con indicadores categóricos, se aplicó el estimador weighted least squares with mean- and variance-adjusted standard errors (WLSMV), recomendado para cuando se modelan variables ordinales-categóricas (Rhemtulla et al., 2012). 
Tabla 1. Equivalencias entre los ítems originales y adaptados del PANSI

PANSI versión colombiana $\quad$ PANSI versión peruana cumplir con lo que otras personas esperaban de ti?

2. ¿Has sentido que tenías el control de la mayoría de las 14. ¿Has sentido que tenías el control de la mayoría de las situaciones de tu vida? situaciones de tu vida?

3. ¿Pensaste en matarte porque no tenías esperanza en el futuro? $\quad 6$ ¿Pensaste en el futuro?

4. ¿Te sentiste tan triste por tu relación con alguien importante, 8. ¿Te sentiste tan triste por tu relación con alguien que quisiste estar muerto?

importante, que quisiste porque no pudiste hacer algo

5. ¿Pensaste en matarte porque no pudiste hacer algo que era 1 ¿ ¿Pensaste en muy importante en tu vida? que era muy importante para ti?

6. ¿Tuviste esperanza en el futuro porque las cosas estaban 11. ¿Tuviste esperanza en el futuro porque las cosas saliendo como tu querías? estaban saliendo como tú querías?

7. ¿Pensaste en matarte porque no encontraste una solución a un 3. ¿Pensaste en problema personal?

solución a un problema personal?

8. ¿Te sentiste alegre porque te estaba yendo bien en el colegio o 12 . ¿Te sentiste alegre porque te estaba yendo bien en la en el trabajo?

9. ¿Pensaste en matarte porque viste que tu vida era un fracaso? universidad?

4. ¿Pensaste en porque viste que tu vida era un fracaso?

10. ¿Pensaste que tus problemas eran tan graves que la única 2. ¿Pensaste que tus problemas eran tan graves que la opción que tenías era suicidarte? única opción que tenías era ?

11. ¿Te sentiste tan solo(a) o tan triste que querías matarte para así terminar con ese sufrimiento?

5. ¿Te sentiste $\tan$ solo(a) o tan triste que querías para así terminar con ese sufrimiento?

12. ¿Tuviste confianza en las capacidades que tenías para enfrentar la mayoría de problemas de tu vida?

13. ¿Sentiste que valía la pena vivir la vida?

9. ¿Tuviste confianza en las capacidades que tenías para enfrentar la mayoría de problemas de tu vida?

13. ¿Sentiste que valía la pena vivir la vida?

14. ¿Tuviste confianza en lograr tus metas en el futuro?

10. ¿Tuviste confianza en lograr tus metas en el futuro?

El ajuste de los modelos factoriales se evaluó a partir de tres índices complementarios: el comparative fit index (CFI), el root mean square error of approximation (RMSEA), y el standardized root mean square residual (SRMR). Valores de CFI mayores o iguales a .90 y .95 han sido sugeridos como indicadores de ajuste a los datos aceptable y excelente respectivamente; mientras que para el caso del RMSEA valores menores que .08 y .05 indican un ajuste a los datos aceptable y excelente en cada caso (Hu \& Bentler, 1999; Marsh et al., 2004; Schreiber et al., 2006). Para el caso del RMSEA se estimó además el intervalo de confianza al $90 \%$. En cuanto al SRMR, valores menores o iguales a .08 son indicadores de un buen ajuste a los datos ( $\mathrm{Hu} \&$ Bentler, 1999; Schreiber, 2017). Con el objetivo de comparar el ajuste relativo de los modelos, se aplicó la prueba de comparación del chi cuadrado con la corrección de segundo orden T3 (Asparouhov \& Muthén, 2006). Esta última corrección es apropiada cuando se utiliza el método de estimación WLSMV. Los análisis CFA, así como las pruebas de diferencias entre modelos, fueron llevados a cabo mediante el software Mplus v.8.5.

Acerca de la confiabilidad, se evalúa el alfa, para comparar los resultados con los antecedentes, y el omega, debido a que es recomendado en estudios que emplean el AFC (Green \& Yang, 2008; Ventura-León \& Caycho-Rodríguez, 2017).

\section{Aspectos éticos}

Por un lado, se siguieron los principios éticos de la Asociación Americana de Psicología, relacionadas al consentimiento, al conocimiento de la temática evaluada, a la posibilidad de declinar de la investigación y la comunicación con los autores. Por otro lado, las directrices de la Comisión Internacional de los Tests (Muñiz et al., 2013), relacionadas con el permiso para adaptar el instrumento la propiedad intelectual del instrumento y a los procedimientos racionales y empíricos del proceso metodológico.

\section{Resultados}

\section{Adaptación del instrumento}

En la Tabla 1 se pueden observar los ítems de la versión colombiana y peruana. En la versión peruana, los ítems fueron presentados agrupados por dimensión; en primer lugar, la dimensión negativa y, en segundo lugar, la positiva; por ello, la numeración es diferente. El ítem 4 original 
Tabla 2. Índices de Ajuste de los modelos CFA

\begin{tabular}{|c|c|c|c|c|c|c|}
\hline Muestra & $\chi^{2}$ & $d f$ & CFI & SRMR & RMSEA $(90 \% \mathrm{CI})$ & $\Delta \chi^{2}$ \\
\hline \multicolumn{7}{|l|}{$\begin{array}{l}\text { Muestra } 1 \\
(\mathrm{~N}=306)\end{array}$} \\
\hline Modelo 1 & 1196.59 & 77 & .911 & .179 & $.218(.207, .229)$ & \\
\hline Modelo 2 & 787.26 & 77 & .943 & .180 & $.174(.163, .185)$ & \\
\hline Modelo 3 & 163.62 & 76 & .993 & .038 & $.061(.048, .074)$ & M3 vs M2 $=88.41^{*}$ \\
\hline \multicolumn{7}{|l|}{$\begin{array}{l}\text { Muestra } 2 \\
(\mathrm{~N}=207)\end{array}$} \\
\hline Modelo 1 & 927.80 & 77 & .890 & .148 & $.231(.218, .244)$ & \\
\hline Modelo 2 & 605.39 & 77 & .932 & .163 & . $182(.169, .196)$ & \\
\hline Modelo 3 & 171.41 & 76 & .988 & .045 & .078 (.062,.093) & M3 vs M2 $=66.22 *$ \\
\hline
\end{tabular}

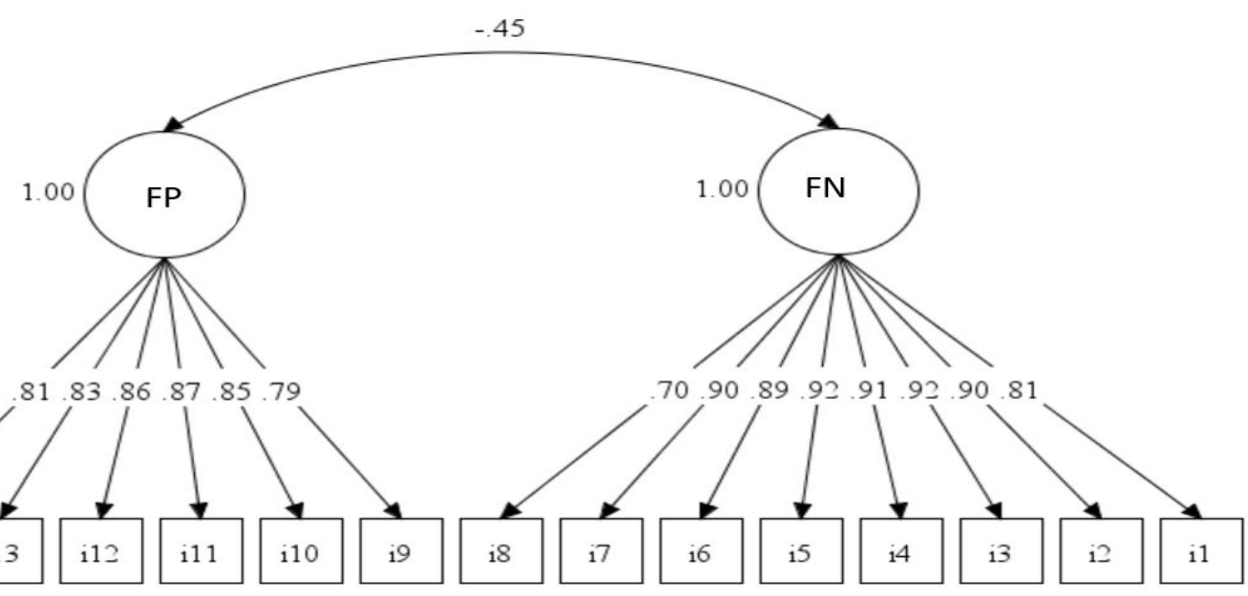

Figura 1. Parámetros Estandarizados del modelo 3. Muestra 1, antes de la pandemia Nota. FN: Factor negativo, FP: Factor positivo

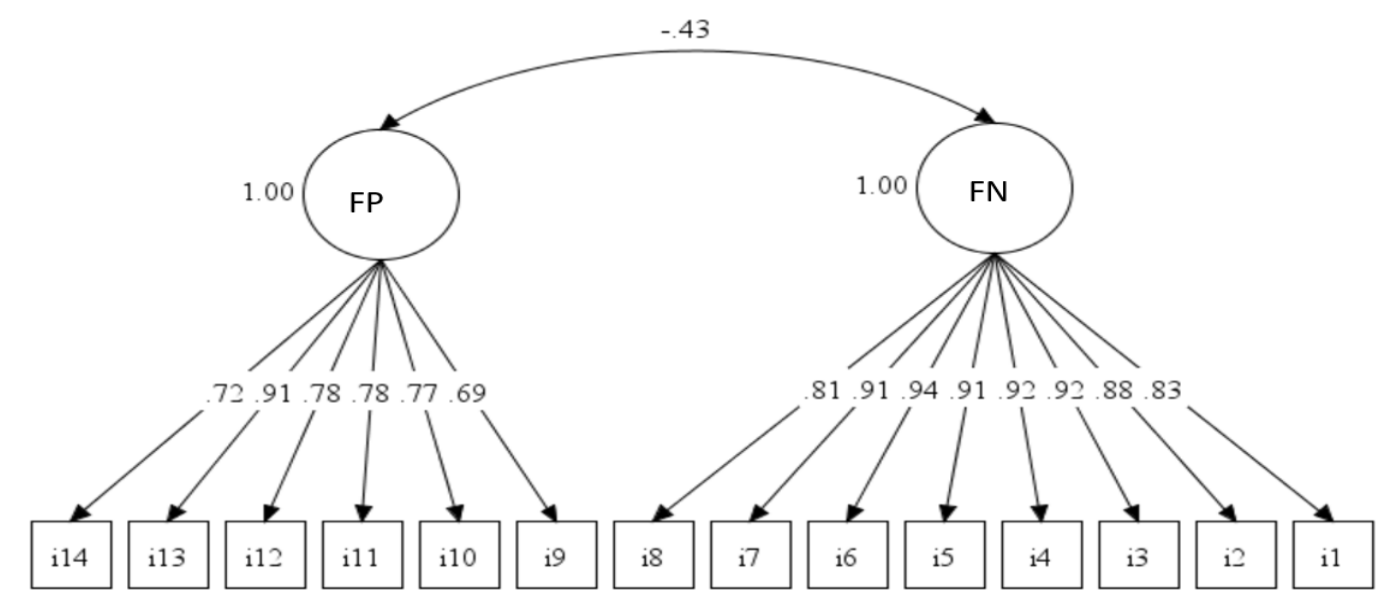

Figura 2. Parámetros Estandarizados del modelo 3. Muestra 2, durante la pandemia Nota. FN: Factor negativo, FP: Factor positivo

indicaba ideación suicida pasiva, mientras que en el presente trabajo fue modificado para que refleje ideación suicida activa, acorde a los demás ítems. El ítem 8 original contemplaba la opción de colegio o trabajo, pero en la versión peruana fue modificado para referir solo al ámbito universitario.

\section{Análisis de Validez Estructural}

En el presente trabajo se pusieron a prueba tres modelos, un modelo unidimensional (Modelo 1), un modelo de dos factores ortogonales (Modelo 2), y el modelo original de dos factores correlacionados (Modelo 3). Los modelos alternativos se agregan com fines comparativos, 
Tabla 3. Confiabilidad de las dimensiones del PANSI

\begin{tabular}{lcccc}
\hline & \multicolumn{2}{c}{$\begin{array}{c}\text { Antes de la } \\
\text { pandemia }\end{array}$} & \multicolumn{2}{c}{$\begin{array}{c}\text { Durante la } \\
\text { pandemia }\end{array}$} \\
\hline & $\begin{array}{c}\text { Factor } \\
\text { negativo }\end{array}$ & $\begin{array}{c}\text { Factor } \\
\text { positivo }\end{array}$ & $\begin{array}{c}\text { Factor } \\
\text { negativo }\end{array}$ & $\begin{array}{c}\text { Factor } \\
\text { positivo }\end{array}$ \\
\cline { 2 - 6 } Alfa & .93 & .91 & .95 & .85 \\
Omega & .96 & .93 & .97 & .90 \\
\hline
\end{tabular}

dado que es posible obtener un ajuste adecuado de un modelo determinado, pero esto no asegura que el ajuste sea mejor a otros modelos plausibles.

A continuación, se describen los resultados obtenidos (Tabla 2). Tanto en la muestra 1 como en la muestra 2 se encuentra evidencia a favor del Modelo 3 de dos factores oblicuos. El Modelo 3 muestra una bondad de ajuste adecuada, siendo las diferencias con el Modelo 2 estadísticamente significativas. En las figuras 1 y 2 se muestran los parámetros estandarizados estimados para el Modelo 3.

\section{Confiabilidad}

Acerca de la consistencia interna, la Tabla 3 revela los adecuados niveles para cada dimensión en ambas muestras. Antes de la pandemia por COVID-19, el Alfa del factor negativo y positivo fueron .93 y .91; mientras que el Omega, .96 y .93 , respectivamente. Durante la pandemia, el Alfa del factor negativo y positivo resultaron $.95 \mathrm{y}$ .85 ; mientras que el Omega, .97 y .90 , respectivamente.

\section{Discusión}

El objetivo del estudio fue analizar las propiedades psicométricas del Inventario de Ideación Suicida Positiva y Negativa (PANSI, Osman et al., 1998) adaptado en universitarios peruanos. Para ello, se partió de la versión colombiana (Villalobos-Galvis, 2010), siguiendo un proceso metodológico lógico-racional y empírico (Muñiz et al., 2013) para lograr el máximo nivel de equivalencia conceptual y cultural.

Se realizaron algunas modificaciones en el fraseo de los ítems, que fueron aprobados por jueces expertos y contrastados mediante entrevista a sujetos de la población, con el fin de atenuar el impacto negativo de los ítems. Dentro del factor ideación suicida negativa, se realizó un cambio conceptual en el ítem cuatro del instrumento original para que se encuentre acorde a los demás ítems de este factor, que miden ideación suicida activa. Esta decisión fue tomada porque el ítem en mención había resultado deficiente en un estudio que empleó la Teoría de Respuesta al Ítem (Avendaño-Prieto et al., 2018). Esta modificación es teórica y empíricamente pertinente, pero futuras investigaciones seguirán analizando su funcionalidad psicométrica. Por otro lado, los ítems del factor ideación suicida positiva quedaron intactos, salvo el ocho de la versión original, que fue modificado para que se refiera solo al ámbito universitario.

Acerca de los índices de ajuste del CFA, cuando se ha comparado un modelo unidimensional frente a uno de dos factores relacionados, el PANSI ha mostrado funcionar adecuadamente con los dos factores (Chang et al., 2009; Muehlenkamp et al, 2005; Osman et al., 1998; Osman et al., 2002; Osman et al., 2003), lo cual es coherente con los resultados presentados. Los demás antecedentes internacionales (Aloba et al., 2018; Sinniah et al., 2015), solo estudiaron el modelo oblicuo de dos factores. La correlación inversa entre factores indica que, a mayor puntuación en ideación suicida negativa, menor será la puntuación en ideación suicida positiva, o viceversa. Esta tendencia también sigue la línea de los estudios previos. Ninguna investigación había evaluado un modelo ortogonal, por lo que fue pertinente examinarlo, concluyendo que no presenta un adecuado ajuste estadístico. Finalmente, la confiabilidad de cada factor refleja apropiada consistencia interna, lo cual coincide con todos los antecedentes.

En base a estas evidencias, se considera que además de la equivalencia conceptual y cultural, también se ha logrado una equivalencia psicométrica, dado que se han encontrado resultados muy parecidos a los antecedentes, en ambas muestras de estudio, antes y durante la pandemia por COVID-19; sin embargo, para incrementar evidencias de validez, es necesario continuar con el estudio psicométrico de esta versión del PANSI, indagando sobre la validez en base a la relación con otros instrumentos y explorando la invarianza de la medición.

Teóricamente, es importante señalar que desde el origen del PANSI (Osman et al., 1998), 
se han distinguido dos factores, uno de pensamientos de riesgo (negativo) y otro de protección (positivo), frente al suicidio; no obstante, debe indicarse que el factor negativo, contiene ítems sobre ideación suicida activa, que debe ser considerado al momento de realizar las interpretaciones cuando se emplea este instrumento. Con esta aclaración, futuros estudios podrían construir una dimensión adicional de ideación suicida pasiva, para integrar estas dos formas de expresión de la ideación suicida y así se pueda abarcar más este constructo.

Del mismo modo, debido a la confusión que podría ocasionar el término ideación suicida positiva, se propone renombrar las dimensiones. Así, la primera podría denominarse ideación suicida; mientras que el segundo, factores protectores frente a la ideación suicida. La primera quedaría redefinida como pensamientos negativos activos relacionados al suicidio, que funcionan como un factor de riesgo para el suicidio; la segunda, como aspectos positivos que funcionan como un factor protector frente al suicidio. En este segundo factor, la cualidad de "pensamientos" tendría que ser criticado porque sus ítems no reflejan ello. Por ejemplo, los ítems 9 (¿Tuviste confianza en las capacidades que tenías para enfrentar la mayoría de problemas de tu vida?) y 10 (¿Tuviste confianza en lograr tus metas en el futuro?) miden autoeficacia; el ítem 11 (¿Tuviste esperanza en el futuro porque las cosas estaban saliendo como tú querías?), refleja esperanza; el ítem 12 ( Te sentiste alegre porque te estaba yendo bien en la universidad?), indica alegría o felicidad; el ítem 13 (¿Sentiste que valía la pena vivir la vida?), sentido de vida; y el ítem 14 (¿Has sentido que tenías el control de la mayoría de las situaciones de tu vida?), autodeterminación. Sin embargo, esto no quita valor teórico al instrumento; todo lo contrario, revela que la psicología positiva puede brindar bases conceptuales para afrontar el suicidio, lo cual amerita futuras discusiones teóricas.

En conclusión, para las muestras estudiadas y pese a las limitaciones del muestreo y el tamaño de las muestras, la versión peruana del PANSI obtuvo adecuadas propiedades psicométricas para un modelo de dos factores relacionados y puede emplearse en estudios multivariados y generar datos que contribuyan con la prevención del suicidio, aportando a los esfuerzos científicos de la región (Benavides-Mora, Villota-Melo, \& Villalobos-Galvis, 2019; Flores-Kanter, 2017; Flores-Kanter, García-Batista, Moretti， \& Medrano, 2019).

\section{Referencias}

Alarcón, R. (2009). Psicología positiva: Un enfoque emergente. Temática Psicológica, 4(1), 7-20.

http://revistas.unife.edu.pe/index.php/tematica psicologica/article/view/881

Aloba, O., Adefemi, S., \& Aloba, T. (2018). Positive and Negative Suicide Ideation (PANSI) Inventory: Initial psychometric properties as a suicide risk screening tool among Nigerian university students. Clinical Medicine Insights: Psychiatry, 9, 1-9. https://doi.org/10.1177/1179557317751910

Asparouhov, T., \& Muthén, B. (2009). Exploratory structural equation modeling. Structural Equation Modeling: A Multidisciplinary Journal, 16(3), 397-438. https://doi.org/10.1080/10705510903008204

Ato, M., López-García, J. J., \& Benavente, A. (2013). Un sistema de clasificación de los diseños de investigación en psicología. Anales de Psicología/Annals of Psychology, 29(3), 1038-1059.

https://doi.org/10.6018/analesps.29.3.178511

Avendaño-Prieto, B., Pérez-Prada, M., ViancháPinzón, M., Martínez-Vaquero, L., \& Toro, R. (2018). Propiedades psicométricas del inventario de ideación suicida positiva y negativa PANSI. Revista Evaluar, 18(1), 27-39. http://dx.doi.org/10.35670/16674545.v18.n1.1 9767

Baader, M. T., Urra, P. E., Millán, A. R., \& Yáñez, M. L. (2011). Algunas consideraciones sobre el intento de suicidio y su enfrentamiento. Revista Médica Clínica Las Condes, 22(3), 303-309. https://doi.org/10.1016/S07168640(11)70430-8

Beck, A. T., Brown, G. K., \& Steer, R. A. (1997). Psychometric characteristics of the Scale for Suicide Ideation with psychiatric outpatients. Behaviour Research and Therapy, 35(11), 1039-1046. https://doi.org/10.1016/S0005-7967(97)00073-9 
Beck, A. T., Kovacs, M., \& Weissman, A. (1979). Assessment of suicidal intention: The Scale for Suicide Ideation. Journal of Consulting and Clinical Psychology, 47(2), 343-352.

https://psycnet.apa.org/doi/10.1037/0022006X.47.2.343

Benavides-Mora, V., Villota-Melo, N. \& Villalobos-Galvis, F. (2019). Conducta suicida en Colombia: Una revisión sistemática. Revista de Psicopatología y Psicología Clínica, 24(3), 181- 195. doi:10.5944/rppc. 24251.

Carmona, J. A., Carmona, D. E., Maldonado, N., Rivera, C., Fernández, O. L., Cañón, S. C., Alvarado, S. V, Jaramillo, J. C., Narváez, M., Fandiño, D. C., Vélez, D., \& Velásquez, H. J. (2017). El suicidio y otros comportamientos autodestructivos en jóvenes universitarios de Colombia y Puerto Rico: Acciones, interacciones y significaciones. Universidad de Manizales: Colombia.

Chang, H. J., Lin, C. C., Chou, K. R., Ma, W. F., \& Yang, C. Y. (2009). Chinese version of the positive and negative suicide ideation: instrument development. Journal of advanced nursing, 65(7), 1485-1496.

https://doi.org/10.1111/j.1365-

2648.2009.05005.x

Chaparro-Narváez, P., Díaz-Jiménez, D., \& Castañeda-Orjuela, C. (2018). Tendencia de la mortalidad por suicidio en áreas urbanas y rurales de Colombia, 19792014. Biomédica, 39(2).

https://doi.org/10.7705/biomedica.v39i3.4427

Denis-Rodríguez, E., Barradas, M. E., DelgadilloCastillo, R., Denis-Rodríguez, P., \& MeloSantisteban, G. (2017). Prevalencia de la ideación suicida en estudiantes de Medicina en Latinoamérica: Un meta análisis. Revista Iberoamericana para la Investigación y el Desarrollo Educativo, 8(15), 387-418. https://doi.org/10.23913/ride.v8i15.304.

Flores Kanter, P. E. (2017). El lugar de la psicología en las investigaciones empíricas del suicidio en Argentina: Un estudio bibliométrico. Interdisciplinaria: Revista de Psicología y Ciencias Afines, 34(1). https://doi.org/10.16888/interd.2017.34.1.2
Flores-Kanter, P. E., García-Batista, Z. E., Moretti, L. S., \& Medrano, L. A. (2019). Towards an explanatory model of suicidal ideation: the effects of cognitive emotional regulation strategies, affectivity and hopelessness. The Spanish Journal of Psychology, 22, E43, 1-11.

doi:10.1017/sjp.2019.45

Goldney, R. D. (2008). Suicide prevention. Retrieved from https://ebookcentral.proquest.com

Gómez, G. A. (2012). Evaluación del riesgo de suicidio: enfoque actualizado. Revista Médica Clínica Las Condes, 23(5), 607-6015. doi.org/10.1016/S0716-8640(12)70355-3

Gómez, R. (2017). Proceso de duelo: Meta análisis del enfrentamiento a la muerte suicida desde una perspectiva familiar. Cuadernos Hispanoamericanos De Psicología, 17(1), 4964. https://doi.org/10.18270/chps.v17i1.2147

González, P., Medina, Ó., \& Ortiz, J. (2016). Riesgo suicida y factores asociados en estudiantes de Psicología en una Universidad pública de Colombia. Revista Habanera de Ciencias Médicas, 15(1), 136-146. https://www.medigraphic.com/cgibin/new/resumen.cgi?IDARTICULO=65072

González-Jaimes, N. L., Tejeda-Alcántara, A. A.., Espinosa-Méndez, C. M., \& OntiverosHernández, Z. O. (2020). Impacto psicológico en estudiantes universitarios mexicanos por confinamiento durante la pandemia por Covid-19.: Psychological impact on Mexican university students due to confinement during the Covid-19 pandemic. https://doi.org/10.1590/SciELOPreprints.756

Green, S. B., \& Yang, Y. (2008). Reliability of summed item scores using structural equation modeling: An alternative to coefficient Alpha. Psychometrika, 74(1), 155-167. doi:10.1007/s11336-008-9099-3

Johnson, M. C., Saletti-Cuesta, L., \& Tumas, N. (2020). Emociones, preocupaciones y reflexiones frente a la pandemia del COVID19 en Argentina. Ciência \& Saúde Coletiva, 25, 2447-2456. https://doi.org/10.1590/141381232020256.1 .10472020

Killgore, W. D. S., Cloonan, S. A., Taylor, E. C., Fernandez, F., Grandner, M. A., \& Dailey, N. 
S. (2020). Suicidal ideation during the COVID-19 pandemic: The role of insomnia. Psychiatry Research, 290, 113-134. https://doi.org/10.1016/j.psychres.2020.113134

Lee, S. A. (2020). Coronavirus anxiety scale: A brief mental health screener for COVID-19 related anxiety. Death Studies, 44(7), 393401. https://doi.org/10.1080/07481187.2020.1 748481

Mamun, M. A., \& Griffiths, M. D. (2020). First COVID-19 suicide case in Bangladesh due to fear of COVID-19 and xenophobia: Possible suicide prevention strategies. Asian Journal of Psychiatry, 51, 102073. https://doi.org/10.1016/j.ajp.2020.102073

Mejia, C. R., Sancho, A. Q., Alarcon, J. F. R., Valero, L. C., López, V. L. P., Villanueva, E. S. V., ... \& Gonzales, J. J. V. (2020). Factores asociados al fatalismo ante la COVID-19 en 20 ciudades del Perú en marzo 2020. Revista Habanera de Ciencias Médicas, 19(2), 8, e3233.

http://scielo.sld.cu/scielo.php?script=sci_artte $\mathrm{xt} \& \mathrm{pid}=\mathrm{S} 1729$ -

519X2020000200015\&lng=es\&tlng=en

Ministerio de Salud. (2018). Lineamientos de Política Sectorial en Salud Mental Perú 2018. Recuperado de: bvs.minsa.gob.pe/local/MINSA/4629.pdf

Muehlenkamp, J. J., Gutierrez, P. M., Osman, A., \& Barrios, F. X. (2005). Validation of the Positive and Negative Suicide Ideation (PANSI) Inventory in a diverse sample of young adults. Journal of clinical psychology, 61(4), 431-445. https://doi.org/10.1002/jclp.20051

Muñiz, J., Elosua, P., \& Hambleton, R. K. (2013). Directrices para la traducción y adaptación de los tests: Segunda edición. Psicothema, 25(2), 151-157. 10.7334/psicothema2013.24

Ondé, D. (2020). Revisión del Concepto de Causalidad en el Marco del Análisis Factorial Confirmatorio. Revista Iberoamericana de Diagnóstico y Evaluación - e Avaliação Psicológica, 1(54), 103-118. https://doi.org/10.21865/RIDEP54.1.01

Organización Mundial de la Salud. (2006). Prevención del suicidio. Un instrumento en el trabajo. Recuperado www.who.int/mental_health/prevention/suicid e/resource_work_spanish.pdf

Organización Mundial de la Salud. (2014). Prevención del suicidio, un imperativo global. Resumen ejecutivo. Recuperado de: www.who.int/mental_health/suicideprevention/exe_summary_spanish.pdf?ua=1

Organización Mundial de la Salud. (2019). Suicidio. Nota de prensa 2 de setiembre de $2019 . \quad$ Recuperado de: https://www.who.int/es/news-room/factsheets/detail/suicide

Organización Mundial de la Salud (2020a). COVID-19: cronología de la actuación de la OMS. Recuperado de: https://www.who.int/es/news-room/detail/2704-2020-who-timeline---covid-19

Organización Mundial de la Salud (2020b). Los nombres de la enfermedad por coronavirus (COVID-19) y del virus que la causa. Recuperado de: https://www.who.int/es/emergencies/diseases/ novel-coronavirus-2019/technicalguidance/naming-the-coronavirus-disease(covid-2019)-and-the-virus-that-causes-it

Organización Panamericana de la Salud (2018). La carga de los trastornos mentales en la Región de las Américas, 2018. Washington, D.C.

Osman, A., Barrios, F. X., Gutierrez, P. M., Wrangham, J. J., Kopper, B. A., Truelove, R. S., \& Linden, S. C. (2002). The Positive and Negative Suicide Ideation (PANSI) inventory: Psychometric evaluation with adolescent psychiatric inpatient samples. Journal of Personality Assessment, 79(3), 512-530. https://doi.org/10.1207/S15327752JPA7903_07

Osman, A., Gutierrez, P. M., Kopper, B. A., Barrios, F. X., \& Chiros, C. E. (1998). The Positive and Negative Suicide Ideation Inventory: Development and validation. Psychological Reports, 82(3), 783793. https://doi.org/10.2466\%2Fpr0.1998.82.3 .783

Osman, A., Gutierrez, P. M., Jiandani, J., Kopper, B. A., Barrios, F. X., Linden, S. C., \& Truelove, R. S. (2003). A preliminary validation of the Positive and Negative Suicide Ideation (PANSI) inventory with normal adolescent samples. Journal of 
Clinical Psychology, 59(4), 493512. https://doi.org/10.1002/jclp.10154

Ozamiz-Etxebarria, N., Dosil-Santamaria, M., Picaza-Gorrochategui, M., \& IdoiagaMondragon, N. (2020). Niveles de estrés, ansiedad y depresión en la primera fase del brote del COVID-19 en una muestra recogida en el norte de España. Cadernos de Saúde Pública, 36, e00054020. https://doi.org/10.1590/0102-311X00054020

Plutchik, R., Van Praag, H. M., Conte, H. R., \& Picard, S. (1989). Correlates of suicide and violence risk 1: The suicide risk measure. Comprehensive Psychiatry, 30(4), 296-302.

https://doi.org/10.1016/0010-440X(89)90053-9

Pompili, M. (Ed.). (2012). Suicide: A global perspective. Retrieved from https://ebookcentral.proquest.com

Posner, K., Oquendo, M. A., Gould, M., Stanley, B., \& Davies, M. (2007). Columbia Classification Algorithm of Suicide Assessment (C-CASA): Classification of suicidal events in the FDA's pediatric suicidal risk analysis of antidepressants. The American Journal of Psychiatry, 164(7), 1035-1043. https://doi.org/10.1176/ajp.2007.164.7.1035

Quagliata, S. (2015). Las características del duelo en madres de hijos fallecidos por suicidio: estudio de casos. Tesis para obtener el grado de magister en psicología clínica. Universidad de la República, Uruguay, https://hdl.handle.net/20.500.12008/7570

Roberts, R. K., Roberts, C. R., \& Chen, Y. R. (1998). Suicidal thinking among adolescents with a history of attempted suicide. Journal of the American Academy of Child \& Adolescent Psychiatry, 37(12), 1294-1300.

https://doi.org/10.1097/00004583-19981200000013

Rush, A. J., \& Beck, A. T. (1978). Cognitive therapy of depression and suicide. American Journal of Psychotherapy, 32(2), 201-219. https://doi.org/10.1176/appi.psychotherapy.19 78.32.2.201

Sánchez, R., Cáceres, H., \& Gómez, D. (2002). Ideación suicida en universitarios: prevalencia y factores asociados. Biomédica, 22 (Sup2), 407-416.
https://doi.org/10.7705/biomedica.v22iSupp2. 1189

Sánchez-Martí, A., Muñoz, J. L., \& Ion, G. (2019). Diseño y validación de un Cuestionario de Percepción del Aprendizaje a través del feedback entre iguales en educación superior. Revista Iberoamericana de Diagnóstico y Evaluación - e Avaliação Psicológica, 4(53), 113-128.

https://doi.org/10.21865/RIDEP53.4.0

Santana-Campas, M. A., \& Santoyo Telles, F. (2018). Propiedades psicométricas de la escala riesgo suicida de Plutchik en una muestra de jóvenes mexicanos privados de la libertad. Avances en Psicología, 26(1), 57-64. https://doi.org/10.33539/avpsicol.2018.v26n2. 1127

Seligman, M. (2017). La auténtica felicidad. Barcelona. España: Ediciones B.

Seligman, M. E. P. \& Csikszentmihalyi, M. (2014) Positive Psychology: An Introduction. In: Flow and the foundations of positive psychology. Springer, Dordrecht

Sinniah, A., Oei, T. P., Chinna, K., Shah, S. A., Maniam, T., \& Subramaniam, P. (2015). Psychometric Properties and Validation of the Positive and Negative Suicide Ideation (PANSI) Inventory in an Outpatient Clinical Population in Malaysia. Frontiers in Psychology, 6, 1934.

https://doi.org/10.3389/fpsyg.2015.01934

Steenblock, C., Todorov, V., Kanczkowski, W., Eisenhofer, G., Schedl, A., Wong, M.-L., ... Bornstein, S. R. (2020). Severe acute respiratory syndrome coronavirus 2 (SARS$\mathrm{CoV}-2)$ and the neuroendocrine stress axis. Molecular Psychiatry, 25, 1611-1617. https://doi.org/10.1038/s41380-020-0758-9

Tomicic, A., Gálvez, C., Quiroz, C., Martínez, C., Fontbona, J., Rodríguez, J., Aguayo, F., Rosenbaum, C., \& Leyton, F. (2016). Suicide in lesbian, gay, bisexual and trans populations: Systematic review of a decade of research (2004-2014). Revista Médica de Chile, 144(6), 723-733.

https://dx.doi.org/10.4067/S003498872016000600006

Ventura-León, J. L., \& Caycho-Rodríguez, T. (2017). El coeficiente Omega: Un método alternativo para la estimación de la 
confiabilidad. Revista Latinoamericana de Ciencias Sociales, Niñez y Juventud, 15(1), 625-627.

http://www.redalyc.org/articulo.oa?id=773496 27039

Villalobos-Galvis, F. H. (2010). Validez y fiabilidad del Inventario de Ideación Suicida Positiva y Negativa-PANSI, en estudiantes colombianos. Universitas Psychologica, 9(2), 509-520.

https://doi.org/10.11144/Javeriana.upsy9-

2.vfii

Wang, C., Pan, R., Wan, X., Tan, Y., Xu, L., Ho, C. S., \& Ho, R. C. (2020). Immediate psychological responses and associated factors during the initial stage of the 2019 coronavirus disease (COVID-19) epidemic among the general population in China. International Journal of Environmental Research and Public Health, 17(5), 1729. https://doi.org/10.3390/ijerph17051729

Sher, L. (2020). COVID-19, anxiety, sleep disturbances and suicide. Sleep Medicine, 70, 124. https://doi.org/10.1016/j.sleep.2020.04.0 19

Thakur, V., \& Jain, A. (2020). COVID 2019suicides: A global psychological pandemic. Brain, behavior, and immunity, 88 , 952-953.

https://doi.org/10.1016/j.bbi.2020.04.062

Toro-Tobar, R. A., Grajales-Giraldo, F. L., Sarmiento-López, J. C. (2016). Riesgo suicida según la tríada cognitiva negativa, ideación, desesperanza y depresión. Aquichan, 16(4), 473-486.

http://dx.doi.org/10.5294/aqui.2016.16.4.6 